\title{
Tensões de crescimento longitudinais e propriedades mecânicas da madeira de clones de Eucalyptus spp.
}

\author{
Longitudinal growth stress and mechanical \\ properties of wood from Eucalyptus spp. \\ clones
}

\footnotetext{
${ }^{1}$ Programa de Pós-Graduação em Ciência e Engenharia de Materiais, Universidade Federal de Pelotas, Pelotas, RS e-mail: Rafael.beltrame@yahoo.com.br; matheusldeperes@gmail.com; r.delucis@hotmail.com; darcigatto@yahoo.com

${ }^{2}$ Programa de Pós Graduação em Engenharia Florestal, Universidade Federal de Santa Maria, Santa Maria, Brasil. e-mail: leaodanilo@hotmail.com; clovis.haselein@ufsm.br
}

\section{RESUMO}

Em vista da crescente utilização da madeira de eucalipto no setor industrial e da frequência com que ocorrem defeitos de crescimento e secagem, se devem ao fator espécie. O presente estudo objetivou analisar características tecnológicas da madeira de clones do gênero Eucalyptus. Foram selecionados 29 clones aos três e sete anos de idade no município de Tapes, Rio Grande do Sul. Nas árvores em pé e vivas, foi avaliada a deformação residual longitudinal (DRL) por meio de extensômetro CIRAD - Forêt, bem como a velocidade de propagação de ondas de ultrassom (Vsom), parâmetros os quais originaram resultados de Tensão de Crescimento Longitudinal (TCL). Foram confeccionados corpos de prova para a avaliação das propriedades físicas da madeira como: massa específica básica e básica ponderada e velocidade de propagação de ondas de ultrassom. Também propriedades mecânicas de resistência e rigidez, obtidas pelos ensaios de flexão estática, compressão paralela às fibras, cisalhamento e dureza Janka, sendo que para os ensaios de propagação de onda ultrassonora e de flexão estática, foram propostos dois teores de umidades distintos (12\% e umidade de saturação). Foram calculados os módulos de elasticidade dinâmicos pela técnica ultrassonora para as duas condições de umidade, sendo que os parâmetros mecânicos avaliados na condição de saturação também foram empregados no cálculo da tensão de crescimento longitudinal (TCL1 e TCL2). Em relação à massa especifica e às propriedades mecânicas, destacaram-se os clones 1 (Eucalyptus urophylla x Eucalyptus maidenii), 23 (Eucalyptus saligna x Eucalyptus saligna) e 24 (Eucalyptus saligna x Eucalyptus saligna). Já do ponto de vista dos níveis de DRL, ressaltaram-se os clones 68 (Eucalyptus urophylla x Eucalyptus globulus) e 72 (Eucalyptus urophylla x Eucalyptus globulus), os quais podem ser indicados para o melhoramento genético tendo em vista à propensão a incidência de defeitos oriundos das tensões de crescimento.

Palavras-chave: deformações, massa específica básica ponderada, qualidade da madeira, modulo de elasticidade dinâmico.

\section{ABSTRACT}

With the increasing use of eucalypt wood in the industrial sector and against the high occurrence of growth defects and drying, which are due to the specie factor. The present study aimed to analyze technological characteristics of clones from Eucalyptus genus. 29 clones with three and seven years were selected in Tapes, state of Rio Grande do Sul. Longitudinal residual strain (LRS) in standing and vivid trees was evaluated using an extensometer CIRAD - Forêt and an ultrasound to obtain the velocity of propagation of ultrasound waves (Vsom), parameters that were used to determine longitudinal growth stress (LGS). Samples were prepared to determine the physical properties: basic specific gravity, weighed basic specific gravity and velocity of propagation of ultrasound waves. Strength and stiffness at static bending, compression parallel to the grain and Janka hardness tests were determined. Two different levels of moisture content (12\% and saturation) for 
ultrasound and static bending tests e testing were proposed. Dynamic modulus of elasticity by ultrasound technique were determined for the two moisture conditions; and the mechanical parameters evaluated at saturation condition were also used in the determination of the longitudinal growth stress (LGS1 and LGS2). Regarding to the specific gravity and mechanical properties, the highlights were clone 1 (Eucalyptus urophylla $\mathrm{x}$ Eucalyptus maidenii), clone 23 (Eucalyptus saligna x Eucalyptus saligna) and clone 24 (Eucalyptus saligna $\mathrm{x}$ Eucalyptus saligna). From the point of view of the levels of DRL, clone 68 (Eucalyptus urophylla x Eucalyptus globulus) and clone 72 (Eucalyptus urophylla x Eucalyptus globulus) may be suitable for breeding due to the tendency of the incidence of defects from the growth stresses.

Keywords: deformations, basic density weighted, wood quality, dynamic modulus of elasticity.

\section{INTRODUÇÃO}

O gênero Eucalyptus apresenta destaque no setor madeireiro por apresentar atributos que o tornam uma importante fonte de matéria-prima fabril, entre os quais, destacam-se sua capacidade produtiva e sua adaptabilidade a diversos ambientes, o que possibilita que as madeiras das espécies desse gênero atendam satisfatoriamente a diversos requisitos tecnológicos [1].

Historicamente, os povoamentos já implantados no Brasil foram conduzidos visando à produção de celulose e papel. Secundariamente, os genótipos que apresentaram as maiores taxas de crescimento e forma florestal foram selecionados para o emprego na confecção de produtos sólidos. Dessa maneira, parâmetros importantes como os níveis de tensões de crescimento foram subestimados, o que acarretou na atual problemática da elevada frequência que ocorrem má formação de peças de madeira em procedimentos manufatureiros, como por exemplo, a produção de madeira serrada [2].

O resultado dos elevados níveis de tensões de crescimento reflete-se na diminuição do rendimento em serrarias, entre outras inconformidades na obtenção de peças, como os empenamentos, culminando no aumento dos custos de produção. Assim, esses efeitos negativos limitam a utilização da madeira do gênero Eucalyptus para a produção de madeira serrada de qualidade [3].

Os defeitos decorrentes da liberação das tensões de crescimento podem ser elencados como: as fendas e rachaduras nos extremos das toras, os empenamentos e rachaduras longitudinais durante o desdobro de toras e pranchões e o cerne quebradiço, devido à excessiva tensão de compressão nas proximidades da medula [4]. Entretanto, no desenvolvimento das árvores, as tensões de crescimento têm papel importante em sua sustentação, propiciando a rigidez estrutural requerida perante a ação de ventos fortes, pelo peso de sua copa, bem como às inclinações de natureza topográfica.

Nesse contexto, o melhoramento genético aplicado ao gênero Eucalyptus apresenta-se como uma opção potencial para o aumento da produção de madeira. Isso é possível não apenas pela capacidade produtiva e pela alta adaptabilidade das espécies desse gênero, mas, principalmente, pela variabilidade inter e intraespecífica, que expressam diversas propriedades anatômicas, físicas, mecânicas e químicas da madeira, tornando possível atender aos requisitos tecnológicos dos mais variados setores da produção madeireira e industrial [5].

Portanto, para uma classificação efetiva de genótipos superiores do gênero Eucalyptus para fins de seleção genética, um programa de melhoramento deve ser proposto com base em diversas propriedades da madeira, utilizando diversas técnicas de avaliação em consórcio [6]. Dentre as muitas propriedades da madeira existentes, os níveis de tensão de crescimento, estimados pela deformação residual longitudinal têm sido ressaltados no meio cientifico por se tratar de um parâmetro de fácil e rápida mensuração. Ao mesmo tempo, também é confiável com vistas à seleção de árvores menos propensas a apresentar defeitos na formação de peças sólidas, as quais podem ser indicadas para programas de melhoramento genético [7].

Dada essa problemática da falta de informações efetivas sobre a qualidade da madeira de clones híbridos do gênero Eucalyptus que possibilitem a indicação dos genótipos mais adequados para o emprego em programas de melhoramento genético, o presente estudo teve como objetivo avaliar e discutir as tensões de crescimento e as propriedades mecânicas, bem como a massa específica da madeira de clones do gênero Eucalyptus.

\section{MATERIAIS E MÉTODOS}

\subsection{Procedimento experimental}

Foram selecionados 29 clones de Eucalyptus spp. com três e sete anos de idade, no município de Tapes, Rio Grande do Sul (30³4’34”S, 51³0’50”O), utilizando-se como parâmetros de seleção, o diâmetro a altura do peito (DAP; $1,3 \mathrm{~m}$ ) e altura comercial (altura até a primeira bifurcação) para as árvores de sete anos e o DAP 
para as árvores de três anos. O DAP e a altura comercial foram medidos por meio de uma fita métrica e aparelho Vertex III, respectivamente. A partir dessas propriedades dendrométricas, os clones foram divididos em três grupos utilizando-se o método das k-médias [8, 9] (Tabela 1).

Tabela 1: Descrição dos clones de Eucalyptus spp. selecionados para o estudo.

\begin{tabular}{|c|c|c|c|c|c|}
\hline \multicolumn{2}{|c|}{ GRUPO 1} & \multicolumn{2}{|c|}{ GRUPO 2} & \multicolumn{2}{|c|}{ GRUPO 3} \\
\hline Clone & Genealogia & Clone & Genealogia & Clone & Genealogia \\
\hline 8 & E. urophylla $\times$ E. grandis & 1 & E. urophylla $\times$ E.maidenii & 7 & E. urophylla $\times$ E. grandis \\
\hline 9 & E. urophylla $\times$ E. grandis & 3 & E. globuluss $x$ E. grandis & 16 & E. grandis $x$ E.maidenii \\
\hline 12 & E. grandis $x$ E. grandis & 10 & E. urophylla $\times$ E. grandis & 22 & E. saligna $x$ E. saligna \\
\hline 26 & E. grandis $x$ E. grandis & 11 & E. urophylla $\times$ E. grandis & 23 & E. saligna $x$ E. saligna \\
\hline 29 & E. grandis $x$ E. grandis & 13 & E. urophylla $\times$ E. grandis & & \\
\hline 31 & E. saligna $\times$ E. saligna & 14 & E. urophylla x E.kirtoniana & & \\
\hline 33 & E. urophylla $\times$ E. grandis & 21 & E. urophylla $\times$ E. grandis & & \\
\hline 34 & E. urophylla $\times$ E. grandis & 24 & E. saligna $\times$ E. saligna & & \\
\hline 35 & E. urograndis $x$ E. grandis & 25 & E. urophylla $\times$ E. grandis & & \\
\hline 53 & E. urophylla $\times$ E.globulus & 27 & E. urophylla $\times$ E. grandis & & \\
\hline 54 & \multicolumn{5}{|l|}{ E. urophylla $\times$ E.globulus } \\
\hline 64 & \multicolumn{5}{|l|}{ E. urophylla $\times$ E.globulus } \\
\hline 68 & \multicolumn{5}{|l|}{ E. urophylla $\times$ E.globulus } \\
\hline 72 & \multicolumn{5}{|l|}{ E. urophylla $\times$ E.globulus } \\
\hline 73 & \multicolumn{5}{|l|}{ E. urophylla $\times$ E.globulus } \\
\hline
\end{tabular}

De cada clone, foram analisadas oito árvores, totalizando a amostragem de 232 indivíduos arbóreos. Como critérios de seleção na floresta, foi examinada a forma florestal das árvores, de modo a selecionar árvores com tronco cilíndrico e ausência de bifurcações. Adicionalmente, foram evitadas árvores de bordadura a fim de evitar madeiras com anormalidades de crescimento decorridas da ação de ventos.

Em sequência, a Deformação Residual Longitudinal (DRL) foi medida de maneira indireta nas árvores em pé e vivas por meio de um extensômetro produzido pelo Centre de Coopération Internationale em Recherche Agronomique Pour Le Développement - CIRAD - Fôret em quatro posições distintas ao redor do tronco das árvores, conforme as quatro direções cardinais [7].

Para a avaliação da DRL (Figura 1), realizou-se um descascamento da árvore de modo a abrir-se uma janela ao seu redor na altura do DAP. Posteriormente, com auxílio de um gabarito, foram inseridos dois pinos na direção da grã, distantes entre si verticalmente de $45 \mathrm{~mm}$, tendo esses a finalidade de fixar o aparelho de medição (relógio comparador digital). Em sequência, foi introduzida uma broca de $20 \mathrm{~mm}$ de diâmetro entre os pinos, a uma profundidade de $20 \mathrm{~mm}$. A leitura da DRL consistiu no valor mostrado no visor do aparelho logo após o rompimento do tecido lenhoso entre os pinos, causado pela perfuração da broca. A medida da DRL não foi realizada na presença de ventos. 


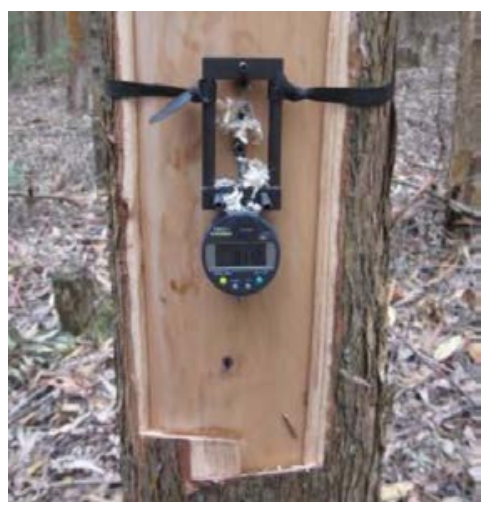

Figura 1: Medição à campo da DRL em árvores em pé e vivas pelo método CIRAD-Forêt.

Aproveitando o descascamento parcial das árvores, foi avaliada a velocidade de propagação de onda ultrassonora (Vsom) utilizando-se um aparelho de ultrassom da marca Proceq dotado de dois transdutores piezelétricos do tipo ponto seco com frequência de excitação de $45 \mathrm{~Hz}$, os quais foram posicionados com auxílio de um gabarito na altura do DAP a uma distância vertical entre si de $30 \mathrm{~cm}$. A partir da velocidade da onda ultrassonora, foi calculado o Módulo de elasticidade dinâmico (Ed1) e a Tensão de crescimento longitudinal (TCL) por meio das Equações 1e 2, respectivamente.

$$
\begin{aligned}
& \mathrm{Ed}_{1}=\mathrm{Vsom} \cdot \rho_{\mathrm{b}} \cdot 10^{-6} \\
& \mathrm{TCL}=\mathrm{Ed} \cdot \mathrm{DRL} \cdot 45^{-1}
\end{aligned}
$$

Em que: Ed1= Módulo de elasticidade dinâmico em campo (MPa); Vsom= Velocidade ultrassonora (m.s-1); TCL= Tensão de crescimento longitudinal em campo (MPa); DRL= Deformação residual longitudinal (mm).

Após a derrubada das árvores, foram retirados discos de $2 \mathrm{~cm}$ de espessura nas posições correspondentes as alturas de 0,1m (base) e do DAP, bem como nas posições de 25, 50, 75 e 100\% da altura comercial. O diâmetro de cada disco foi mensurado sem casca por meio de uma fita métrica com resolução de 1 mm, tendo no topo o valor limite de $10 \mathrm{~cm}$ de diâmetro (Figura 2).
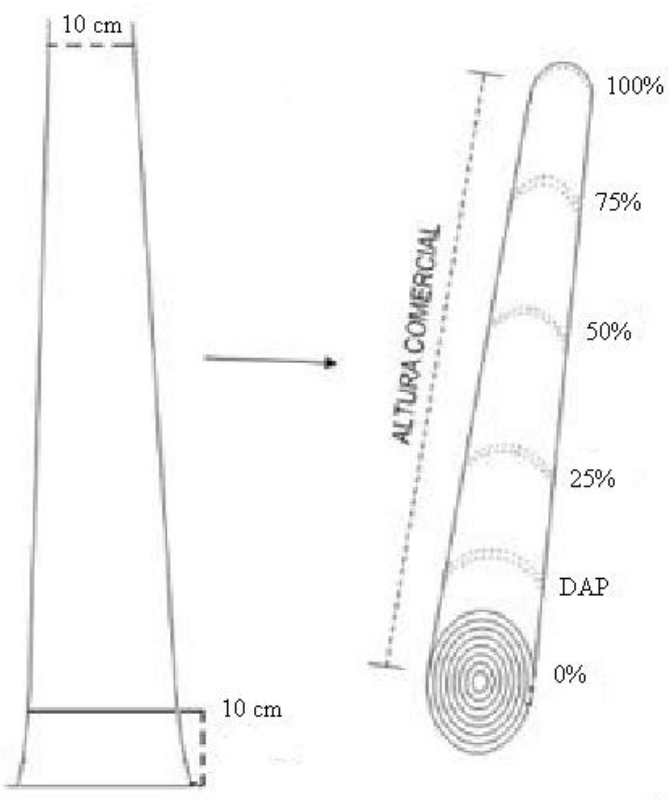

(a)

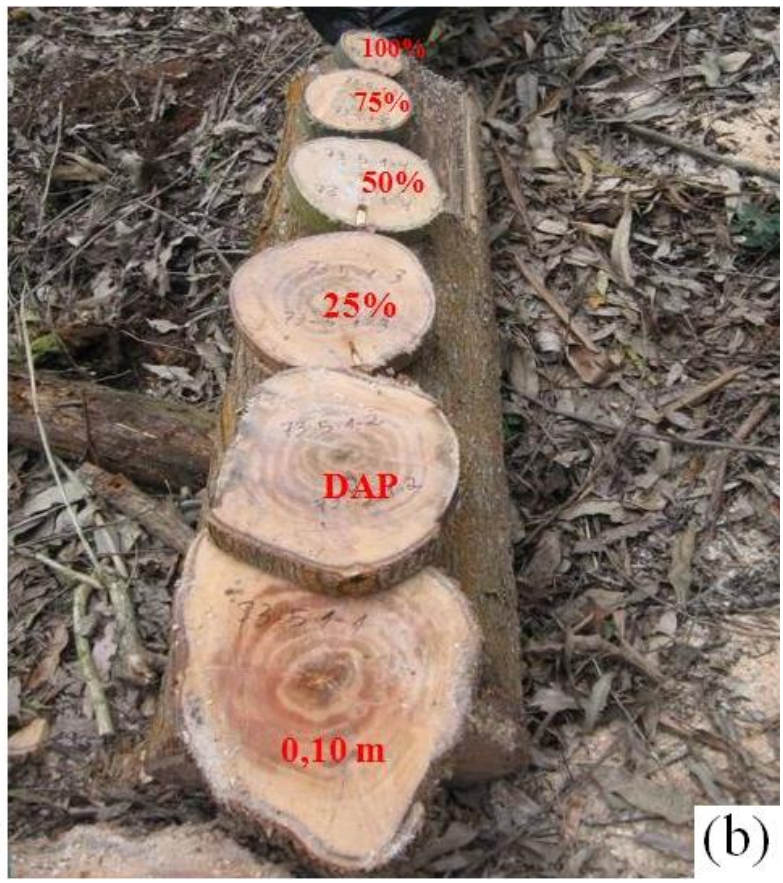

Figura 2: Amostragem dos discos para a determinação da massa específica. (a) representação da posição de retirada dos discos; (b) discos identificados em razão da posição axial de retirada. 
Os discos foram segmentados em duas cunhas simetricamente opostas, as quais foram inseridas em água até atingirem massa constante. A partir dessa condição, foi determinado o volume de cada cunha pelo método de imersão em água sobre balança hidrostática [10]. As cunhas foram submetidas à secagem convencional em estufa laboratorial sob a temperatura de $103 \pm 2{ }^{\circ} \mathrm{C}$ até atingirem massa constante, a partir da qual foram pesadas por meio de uma balança analítica com resolução de 0,01g. A partir desses dados, foram calculadas a massa específica básica e massa específica básica ponderada de cada cunha por meio das equações 3 e 4, respectivamente.

$$
\begin{gathered}
\rho b=m a / V s \\
\rho_{\text {pond }}=\left[\left(\rho_{\mathrm{b} 0 \%}+\rho_{\mathrm{bDAP}}\right) / 2\right] \cdot \mathrm{V}_{1}+\left[\left(\rho_{\mathrm{bn}}+\rho_{\mathrm{b}(\mathrm{n}+1)}\right) / 2\right] \cdot \mathrm{V}_{\mathrm{n}}+\left[\left(\rho_{\mathrm{b} 75 \%}+\rho_{\mathrm{b} 100 \%}\right) / 2\right] \cdot \mathrm{V}_{6}
\end{gathered}
$$

Em que: $\rho b=$ massa específica básica (g.cm-3); ma= massa anidra (g); Vs= volume de saturação (cm3); ppond = massa específica básica ponderada (g.cm-3); $\mathrm{n}=$ posição axial correspondente à retirada da cunha; V1= volume sem casca correspondente às posições 0\% e DAP; V6= volume sem casca correspondente às posições 75 e $100 \%$.

\begin{tabular}{|c|c|c|c|}
\hline Ensaio & Propriedade & Dimensões de CP (cm) & $\begin{array}{l}\text { Velocidade de ensaio } \\
\left(\mathrm{mm}_{\mathrm{min}}^{-1}\right)\end{array}$ \\
\hline \multirow{2}{*}{$\begin{array}{l}\text { Compressão paralela às } \\
\text { fibras }\end{array}$} & $\begin{array}{l}\text { Módulo de elasticidade } \\
\left(\mathrm{Ec}_{12 \%}\right)\end{array}$ & \multirow{2}{*}{$5 \times 5 \times 20$} & \multirow{2}{*}{$e^{2}+a_{1}$} \\
\hline & $\begin{array}{l}\text { Resistência à compressão } \\
\left(\mathrm{fc}_{12 \%}\right)\end{array}$ & & \\
\hline \multirow{3}{*}{ Flexão estática } & $\begin{array}{l}\text { Módulo de elasticidade } \\
\left(\mathrm{MOE}_{12 \%}\right)\end{array}$ & \multirow{3}{*}{$2,5 \times 2,5 \times 41$} & \multirow{3}{*}{1,3} \\
\hline & $\begin{array}{l}\text { Módulo de elasticidade } \\
\text { saturado (MOE) }\end{array}$ & & \\
\hline & $\begin{array}{l}\text { Módulo de ruptura } \\
\left(\mathrm{MOR}_{12 \%}\right)\end{array}$ & & \\
\hline Cisalhamento & $\begin{array}{l}\text { Resistência ao cisalhamen- } \\
\text { to a } 12 \%\end{array}$ & $5,0 \times 5,0 \times 6,25$ & 0,6 \\
\hline Dureza Janka & $\begin{array}{l}\text { Resistência à penetração de } \\
\text { topo a } 12 \%\end{array}$ & $5,0 \times 5,0 \times 15,0$ & 6,0 \\
\hline
\end{tabular}

Tabela 2: Propriedades mecânicas da madeira de Eucalyptus spp.

Para a realização dos ensaios mecânicos, foram selecionadas as toras entre as posições DAP e 25\% da altura comercial, das quais foram desdobrados pranchões com $8 \mathrm{~cm}$ de espessura, centrados pela medula e orientados em relação ao eixo longitudinal das toras. Em sequência, foram seccionados corpos de provas, excluindose a região da medula, os quais após sua confecção foram condicionados ao teor de umidade de $12 \%$ sob as condições de $20 \pm 2^{\circ} \mathrm{C}$ de temperatura e $65 \pm 5 \%$ de umidade relativa. Após, os ensaios mecânicos foram conduzidos de acordo com as diretrizes normativas previstas pelo procedimento D143-94 da American Society for Testing of Materials - ASTM [11] (Tabela 2).

Tabela 2: Propriedades mecânicas da madeira de Eucalyptus spp.

Metade dos corpos de prova selecionados para o ensaio de flexão estática foram imersos em água até a obtenção de massa constante, condição a partir da qual foi avaliada a velocidade de propagação de ondas de ultrassom utilizando-se o aparelho de ultrassom já mencionado, e posteriormente foi calculado o Módulo de elasticidade dinâmico (Ed2) por meio da Equação 1 supracitada. Adicionalmente, a Tensão de crescimento longitudinal (TCL) foi calculada utilizando-se como variáveis independentes o Módulo de elasticidade dinâmico ao ensaio de ultrassom (EdS) e o Módulo de elasticidade estático ao ensaio de flexão estática (MOES) na condição de saturação dos corpos de prova aplicando-se as equações 5 e 6, respectivamente.

$$
\mathrm{TCL}_{1}=\mathrm{Ed} \cdot \mathrm{DRL} \cdot 45^{-1}
$$




$$
\mathrm{TCL}_{2}=\mathrm{MOE} \cdot \mathrm{DRL} \cdot 45^{-1}
$$

Em que: TCL1= Tensão de crescimento longitudinal (método ultrassonoro); TCL2= Tensão de crescimento longitudinal (ensaio de flexão estática); Ed= módulo de elasticidade dinâmico (MPa); MOE= modulo de elasticidade estático obtido pelo ensaio de flexão estática (MPa); DRL= Deformação residual longitudinal (mm).

O Módulo de elasticidade estático - obtido através do ensaio de flexão estática - na condição de saturação foi corrigido conforme o procedimento proposto por BODIG e JAYNE [12], de modo a ser desconsiderado o efeito do esforço de cisalhamento ocorrido no ensaio mecânico.

\subsection{Análise estatística}

Para a análise estatística dos dados obtidos, foi empregado um planejamento experimental inteiramente casualizado, a partir do qual foram aplicadas análises de variância multifatorial tendo como variável independente a $\rho b$ e as propriedades mecânicas e como fatores os clones, os blocos e a posição axial. A ppond, a DRL e as propriedades mecânicas foram desdobradas em razão dos clones por meio de análise de agrupamento Scottknott em nível de probabilidade de erro de 1\%, já a TCL e novamente a ppond foram desdobradas em razão do fator bloco por meio do teste de médias DMS de Fisher em nível de probabilidade de erro de 5\%. Por fim, as relações funcionais entre as variáveis em estudo foram avaliadas por meio do coeficiente de correlação de Pearson.

\section{RESULTADOS E DISCUSSÃO}

\subsection{Massa específica}

Os resultados contidos na Tabela 3 dão conta de que massa específica básica sofreu efeito significativo dos fatores Clone e posição axial, entretanto não significativo do fator Bloco.

Tabela 3: Resumo da análise de variância da massa específica básica $(\rho b)$ e posições na direção axial para os clones de Eucalyptus spp.

\begin{tabular}{l|l|l|l|l|l}
\hline FV & GL & SQ & QM & F & Valor F \\
\hline Clones & 28 & 0,67317 & 0,02404 & 51,78 & $<0,0001^{*}$ \\
\hline Bloco & 1 & 0,00090 & 0,00090 & 1,95 & $0,1638^{\text {NS }}$ \\
\hline Posição & 5 & 0,07695 & 0,01539 & 33,15 & $<0,0001^{*}$ \\
\hline Erro & 313 & 0,14532 & 0,00046 & - & - \\
\hline Total & 347 & 0,896366 & - & - & - \\
\hline
\end{tabular}

Em que: FV = fonte de variação; GL = graus de liberdade; SQ = soma dos quadrados; QM = quadrado médio; F = valor de F calculado; *= significativo em 5\% de probabilidade de erro; NS = não significativo.

$\mathrm{Na}$ comparação dos valores médios de $\rho \mathrm{b}$ entre as posições axiais, verifica-se que a base apresentou o maior valor médio (0,448 g.cm-3), seguida pelas posições 75 e 100\% da altura comercial, ambas com valor médio de 0,442 g.cm-3 (Tabela 4). 
Tabela 4: Valores médios da massa específica básica $(\rho b)$, por posição no sentido axial, e massa específica básica ponderada ( $\rho$ pond), em função do volume comercial sem casca, para cada clone de Eucalyptus spp.

\begin{tabular}{|c|c|c|c|c|c|c|c|c|}
\hline \multirow{2}{*}{ Clones } & \multirow{2}{*}{ Grupos } & \multicolumn{6}{|c|}{ Posição relativa no sentido base-topo } & \multirow{2}{*}{$\begin{array}{l}\rho_{\text {pond }} \\
\left(\mathrm{g} / \mathrm{cm}^{3}\right)\end{array}$} \\
\hline & & $0,1 \mathrm{~m}$ & DAP & $25 \%$ & $50 \%$ & $75 \%$ & $100 \%$ & \\
\hline 8 & 1 & 0,477 & 0,454 & 0,462 & 0,485 & 0,473 & 0,467 & $0,471 \mathrm{c}$ \\
\hline 9 & 1 & 0,398 & 0,353 & 0,370 & 0,382 & 0,390 & 0,393 & $0,377 \mathrm{~g}$ \\
\hline 12 & 1 & 0,394 & 0,338 & 0,345 & 0,384 & 0,367 & 0,376 & $0,363 \mathrm{~g}$ \\
\hline 26 & 1 & 0,442 & 0,351 & 0,353 & 0,366 & 0,386 & 0,410 & $0,368 \mathrm{~g}$ \\
\hline 29 & 1 & 0,458 & 0,390 & 0,380 & 0,393 & 0,425 & 0,424 & $0,398 \mathrm{f}$ \\
\hline 31 & 1 & 0,521 & 0,438 & 0,419 & 0,431 & 0,453 & 0,462 & $0,437 \mathrm{~d}$ \\
\hline 33 & 1 & 0,444 & 0,422 & 0,439 & 0,455 & 0,436 & 0,424 & $0,439 \mathrm{~d}$ \\
\hline 34 & 1 & 0,505 & 0,438 & 0,455 & 0,471 & 0,482 & 0,480 & $0,463 \mathrm{c}$ \\
\hline 35 & 1 & 0,394 & 0,346 & 0,362 & 0,398 & 0,419 & 0,406 & $0,385 \mathrm{f}$ \\
\hline 53 & 1 & 0,418 & 0,370 & 0,380 & 0,406 & 0,411 & 0,424 & $0,396 \mathrm{f}$ \\
\hline 54 & 1 & 0,422 & 0,389 & 0,386 & 0,406 & 0,432 & 0,445 & $0,405 \mathrm{e}$ \\
\hline 64 & 1 & 0,471 & 0,440 & 0,433 & 0,448 & 0,475 & 0,467 & $0,449 \mathrm{c}$ \\
\hline 68 & 1 & 0,408 & 0,402 & 0,415 & 0,438 & 0,428 & 0,460 & $0,425 \mathrm{~d}$ \\
\hline 72 & 1 & 0,427 & 0,380 & 0,372 & 0,401 & 0,417 & 0,435 & $0,395 \mathrm{f}$ \\
\hline 73 & 1 & 0,414 & 0,395 & 0,408 & 0,437 & 0,456 & 0,464 & $0,426 \mathrm{~d}$ \\
\hline 1 & 2 & 0,543 & 0,515 & 0,519 & 0,538 & 0,584 & 0,539 & $0,535 \mathrm{a}$ \\
\hline 3 & 2 & 0,446 & 0,399 & 0,375 & 0,396 & 0,472 & 0,417 & $0,406 \mathrm{e}$ \\
\hline 10 & 2 & 0,426 & 0,401 & 0,413 & 0,422 & 0,425 & 0,407 & $0,415 \mathrm{e}$ \\
\hline 11 & 2 & 0,404 & 0,361 & 0,356 & 0,357 & 0,363 & 0,354 & $0,361 \mathrm{~g}$ \\
\hline 13 & 2 & 0,413 & 0,387 & 0,396 & 0,428 & 0,434 & 0,432 & $0,414 \mathrm{e}$ \\
\hline 14 & 2 & 0,439 & 0,386 & 0,398 & 0,416 & 0,429 & 0,425 & $0,410 \mathrm{e}$ \\
\hline 21 & 2 & 0,424 & 0,383 & 0,393 & 0,403 & 0,406 & 0,411 & $0,398 \mathrm{f}$ \\
\hline 24 & 2 & 0,504 & 0,481 & 0,507 & 0,509 & 0,505 & 0,474 & $0,494 \mathrm{~b}$ \\
\hline 25 & 2 & 0,462 & 0,459 & 0,474 & 0,462 & 0,452 & 0,436 & $0,463 \mathrm{c}$ \\
\hline 27 & 2 & 0,430 & 0,358 & 0,354 & 0,391 & 0,415 & 0,418 & $0,380 \mathrm{~g}$ \\
\hline 7 & 3 & 0,441 & 0,449 & 0,493 & 0,509 & 0,514 & 0,513 & $0,496 \mathrm{~b}$ \\
\hline 16 & 3 & 0,454 & 0,410 & 0,405 & 0,430 & 0,460 & 0,490 & $0,431 \mathrm{~d}$ \\
\hline 22 & 3 & 0,481 & 0,481 & 0,492 & 0,507 & 0,491 & 0,473 & $0,494 \mathrm{~b}$ \\
\hline 23 & 3 & 0,537 & 0,501 & 0,518 & 0,536 & 0,533 & 0,519 & $0,522 \mathrm{a}$ \\
\hline Médias & & 0,448 & 0,409 & 0,416 & 0,433 & 0,442 & 0,442 & 0,428 \\
\hline
\end{tabular}

Em que: Médias seguidas por mesma letra na coluna não diferem entre si pelo teste Scott-Knott em 5\% de probabilidade de erro.

Os resultados de $\rho b$ encontrados na literatura para a madeira de Eucalyptus spp., variam entre os 0,340 g.cm-3, encontrado por LIMA et al. [13], avaliando a madeira de Eucalyptus spp. com 16 anos de idade e os 0,730 g.cm- $^{3}$, encontrados por CAIXETA et al. [6], o que indica que os resultados encontrados no presente estudo para essa propriedade física estão de acordo com resultados de pesquisas já realizadas.

A tendência da variação axial dos valores médios de $\rho b$ indica que houve um decréscimo a partir da base até a posição do DAP e posteriormente um acréscimo no sentido base-topo até a posição de 75\%, a partir da qual houve uma estabilização até a posição de $100 \%$ da altura comercial. Essa tendência não é a mesma encontrada em outros estudos publicados no meio científico.

Em seu estudo, TREVISAN et al. [14] e TREVISAN et al. [15], ambos avaliando a madeira de Eucalyptus grandis aos 18 aos de idade verificaram uma tendência de decréscimo a partir da base até a posição do DAP, posteriormente um acréscimo até a posição de $100 \%$ da altura comercial sem tendência de estabili- 
zação desses valores e sem proporcionalidade entre os acréscimos verificados entre cada posição axial. Segundo autores como SILVA [16], ALZATE et al. [17] e TREVISAN [18], a variabilidade dos valores de $\rho b$ pode ser atribuída à idade, mas principalmente aos fatores ambientais e genéticos.

Em relação à ppond, os clones 1 (E. urophylla x E. maidenii) e 23 (E. saligna x E. saligna) distinguiram-se dos demais obtendo os maiores valores, os quais foram de 0,535 e 0,552 g.cm-3, respectivamente. Os menores valores de $\rho$ pond foram verificados nos clones 9 (E. urophylla x E. grandis), 11 (E. urophylla x E. grandis), 12 (E. grandis x E. grandis), 26 (E. grandis x E. grandis) e 27 (E. urophylla x E. grandis). Dessa maneira, em relação a genealogia, pode-se observar que as espécies E. grandis e E. urophylla estiveram associadas aos menores níveis de $\rho$ pond.

Outros autores obtiveram resultados semelhantes avaliando a $\rho$ pond da madeira de Eucalyptus spp.. Como TREVISAN [18], que pesquisando a ppond para Eucalyptus grandis, aos 14 anos de idade, encontrou valores médios entre 0,392 e 0,434 g.cm- ${ }^{3}$. Já TRUGILHO [3], avaliando 11 clones de Eucalyptus spp., aos 6 anos de idade, obteve valores médios de $\rho$ pond de 0,508 g. $\mathrm{cm}^{-3}$.

Quando comparada entre grupos de clones, verificou-se que o valor de ppond do grupo 3 (0,485 g.cm3) foi significativamente maior do que dos outros dois grupos, sendo que os grupos $1(0,413 \mathrm{~g} . \mathrm{cm}-3)$ e 2 $(0,427$ g.cm-3) exibiram resultados semelhantes, os quais não distinguiram-se estatisticamente.

Diante da variabilidade verificada nos valores obtidos de massa específica da madeira nas comparações propostas entre clones e entre posições axiais, verifica-se a eficácia dessa propriedade na qualificação da madeira do gênero Eucalyptus. Diante disso, ALZATE et al. [17] descreveram que essa propriedade pode ser empregada na definição do uso potencial dos clones, seja como madeira sólida (móveis, esquadrias, tacos, uso estrutural etc.), ou como madeira transformada (celulose e papel, laminação, chapas de fibras, etc.).

\subsection{Propriedades mecânicas}

De acordo com os resultados da análise de variância para as propriedades mecânicas da madeira dos clones de Eucalyptus spp., verificou-se que somente o efeito dos clones foi significativo, ou seja, esses resultados seguiram a mesma tendência verificada para a pb (Tabela 5).

Tabela 5: Análise de variância para propriedades mecânicas a 12\% de umidade

\begin{tabular}{|c|c|c|c|c|c|c|c|}
\hline \multirow{3}{*}{ FV } & \multirow{3}{*}{ GL } & \multicolumn{6}{|c|}{ Quadrados médios (MPa) } \\
\hline & & \multicolumn{2}{|c|}{ Compressão paralela } & \multicolumn{2}{|c|}{ Flexão estática } & \multirow{2}{*}{$\begin{array}{l}\text { Resistência } \\
\text { cisalhamento }\end{array}$} & \multirow{2}{*}{ Dureza Janka } \\
\hline & & Ec & fc & MOE & MOR & & \\
\hline Clone & 28 & $1,44 \times 10^{8 *}$ & $1034,9 *$ & $1,09 \times 10^{8} *$ & $3552,32 *$ & $145,501 *$ & $1829,93^{*}$ \\
\hline Bloco & 1 & $1,27 \times 10^{7 \mathrm{NS}}$ & $28,95^{\mathrm{NS}}$ & $3,61 \times 10^{6 \mathrm{NS}}$ & $1871,21^{\mathrm{NS}}$ & $7,692^{\mathrm{NS}}$ & $0,818^{\mathrm{NS}}$ \\
\hline Erro & 28 & $2,69 \times 10^{7}$ & 58,53 & $1,18 \times 10^{7}$ & 737,53 & 15,09 & 102,39 \\
\hline
\end{tabular}

Em que: Ec= módulo de elasticidade a compressão paralela às fibras; fc= resistência a compressão paralela as fibras; $\mathrm{MOE}=$ módulo de elasticidade a flexão estática; $\mathrm{MOR}=$ módulo de ruptura a flexão estática; $\mathrm{FV}=$ fonte de variação; GL= graus de liberdade; *= significativo em 5\% de probabilidade de erro;NS = não significativo.

Complementando os resultados apresentados na Tabela 5, os dados de propriedades mecânicas da madeira contidos na Tabela 6, dão conta de que o clone 23 (E. saligna x E. saligna) mostrou o maior valor para o Ec (18.852 MPa) e juntamente com o clone 1 (E. urophylla x E. maidenii), exibiu os maiores valores para a fc, valores de 53,64 e 57,71 MPa, respectivamente. Esses valores de Ec e fc podem estar associados à alta $\rho b$ apresentada pelos clones citados para as posições DAP e $25 \%$ da altura comercial. 
BELTRAME,R.; PERES, M.L.; DELUCIS, R.A.; FREITAS, D. L; GATTO, D.A.; HASELEIN, C.R. revista Matéria, v.20, n.4, pp. 1061 - 1074, 2015.

Tabela 6: Descrição das propriedades mecânicas da madeira em razão dos clones de Eucalyptus ssp..

\begin{tabular}{|c|c|c|c|c|c|c|c|}
\hline \multirow{2}{*}{ Clones } & \multirow{2}{*}{ Grupos } & \multicolumn{2}{|c|}{ Compressão paralela } & \multicolumn{2}{|c|}{ Flexão estática } & \multirow{2}{*}{$\begin{array}{l}\text { Resistência } \\
\text { ao cisalha- } \\
\text { mento }\end{array}$} & \multirow{2}{*}{$\begin{array}{l}\text { Dureza } \\
\text { Janka }\end{array}$} \\
\hline & & Ec & fc & MOE & MOR & & \\
\hline 8 & 1 & $\begin{array}{l}14130,0 \\
b\end{array}$ & $50,95 \mathrm{~b}$ & 10867,5 c & 87,31 a & $9,48 \mathrm{~b}$ & $52,21 \mathrm{~b}$ \\
\hline 9 & 1 & 9969,8 c & $36,34 \mathrm{e}$ & $8188,4 \mathrm{~d}$ & $66,31 \mathrm{~b}$ & $6,81 \mathrm{~d}$ & $29,55 \mathrm{e}$ \\
\hline 12 & 1 & 9826,3 c & 35,24 e & $8684,1 \mathrm{~d}$ & $62,79 \mathrm{~b}$ & $6,86 \mathrm{~d}$ & $34,81 \mathrm{~d}$ \\
\hline 26 & 1 & $\begin{array}{l}10778,6 \\
\mathrm{c}\end{array}$ & $39,01 \mathrm{e}$ & $9359,9 \mathrm{~d}$ & $69,72 \mathrm{~b}$ & $5,92 \mathrm{~d}$ & $37,70 \mathrm{c}$ \\
\hline 29 & 1 & $\begin{array}{l}13107,9 \\
b\end{array}$ & $41,45 \mathrm{~d}$ & 10404,3 c & $72,69 \mathrm{~b}$ & 7,97 c & 38,12 c \\
\hline 31 & 1 & $\begin{array}{l}12358,3 \\
c\end{array}$ & $45,49 \mathrm{c}$ & 10283,8 c & 80,20 a & $8,85 \mathrm{~b}$ & $43,04 \mathrm{c}$ \\
\hline 33 & 1 & $9700,3 \mathrm{c}$ & $40,68 \mathrm{~d}$ & 9926,6 c & $77,68 \mathrm{~b}$ & $8,21 \mathrm{c}$ & $40,48 \mathrm{c}$ \\
\hline 34 & 1 & $\begin{array}{l}11077,1 \\
\text { C }\end{array}$ & $46,53 \mathrm{c}$ & 10597,7 c & 77,59 b & $9,07 \mathrm{~b}$ & $41,56 \mathrm{c}$ \\
\hline 35 & 1 & $\begin{array}{l}10566,1 \\
\text { с }\end{array}$ & 36,42 e & 9956,7 c & $64,74 \mathrm{~b}$ & $7,47 \mathrm{c}$ & $36,00 \mathrm{~d}$ \\
\hline 53 & 1 & $9638,1 \mathrm{c}$ & $38,62 \mathrm{e}$ & $8524,4 \mathrm{~d}$ & $64,79 \mathrm{~b}$ & $8,08 \mathrm{c}$ & $38,50 \mathrm{c}$ \\
\hline 54 & 1 & $9797,8 \mathrm{c}$ & $40,36 \mathrm{~d}$ & $8520,6 \mathrm{~d}$ & $70,86 \mathrm{~b}$ & $7,90 \mathrm{c}$ & $36,32 \mathrm{~d}$ \\
\hline 64 & 1 & $\begin{array}{l}10425,4 \\
\text { c }\end{array}$ & $45,64 \mathrm{c}$ & 10050,2 c & 86,91 a & $8,54 \mathrm{c}$ & $39,31 \mathrm{c}$ \\
\hline 68 & 1 & $8693,8 \mathrm{c}$ & $42,24 \mathrm{~d}$ & $9290,3 \mathrm{~d}$ & $76,50 \mathrm{~b}$ & $8,59 \mathrm{c}$ & $41,16 \mathrm{c}$ \\
\hline 72 & 1 & $\begin{array}{l}11305,6 \\
\text { c }\end{array}$ & $42,21 \mathrm{~d}$ & $9133,9 \mathrm{~d}$ & $67,55 \mathrm{~b}$ & $6,63 \mathrm{~d}$ & $32,64 \mathrm{e}$ \\
\hline 73 & 1 & $\begin{array}{l}10617,9 \\
\mathrm{C}\end{array}$ & $43,11 \mathrm{~d}$ & 10907,7 c & 80,42 a & 7,92 c & $38,71 \mathrm{c}$ \\
\hline 1 & 2 & $\begin{array}{l}14535,8 \\
b\end{array}$ & $57,71 \mathrm{a}$ & 16368,7 a & 97,63 a & 10,32 a & $51,26 \mathrm{~b}$ \\
\hline 3 & 2 & $\begin{array}{l}11720,9 \\
\mathrm{C}\end{array}$ & $40,11 \mathrm{~d}$ & $8912,3 \mathrm{~d}$ & $70,73 \mathrm{~b}$ & $7,19 \mathrm{~d}$ & $34,78 \mathrm{~d}$ \\
\hline 10 & 2 & 9958,3 c & $38,32 \mathrm{e}$ & $8929,9 \mathrm{~d}$ & $72,93 \mathrm{~b}$ & $7,86 \mathrm{c}$ & $39,30 \mathrm{c}$ \\
\hline 11 & 2 & 9893,8 c & 37,03 e & 10594,9 c & $73,36 \mathrm{~b}$ & $6,83 \mathrm{~d}$ & $31,36 \mathrm{e}$ \\
\hline 13 & 2 & $\begin{array}{l}10975,7 \\
\text { c }\end{array}$ & $41,49 \mathrm{~d}$ & $9040,7 \mathrm{~d}$ & $70,96 \mathrm{~b}$ & 7,93 c & $35,31 \mathrm{~d}$ \\
\hline 14 & 2 & $9280,7 \mathrm{c}$ & $40,66 \mathrm{~d}$ & $9076,7 \mathrm{~d}$ & $72,53 \mathrm{~b}$ & $7,76 \mathrm{c}$ & $38,35 \mathrm{c}$ \\
\hline 21 & 2 & $\begin{array}{l}13115,1 \\
b\end{array}$ & $43,84 \mathrm{~d}$ & $9503,5 \mathrm{~d}$ & $71,36 \mathrm{~b}$ & $8,11 \mathrm{c}$ & $40,70 \mathrm{c}$ \\
\hline 24 & 2 & $\begin{array}{l}15367,0 \\
\text { b }\end{array}$ & 53,32 b & 13727,6 b & $91,42 \mathrm{a}$ & 10,25 a & $51,78 \mathrm{~b}$ \\
\hline 25 & 2 & $\begin{array}{l}13863,5 \\
b\end{array}$ & $46,44 \mathrm{c}$ & 12492,7 b & 88,26 a & $8,44 \mathrm{c}$ & 39,97 c \\
\hline 27 & 2 & $\begin{array}{l}10421,2 \\
\mathrm{C}\end{array}$ & 36,0 e & 10901,2 c & $72,07 \mathrm{~b}$ & $7,094 \mathrm{~d}$ & $32,89 \mathrm{e}$ \\
\hline 7 & 3 & $9646,5 \mathrm{c}$ & $42,09 \mathrm{~d}$ & $10731,9 \mathrm{c}$ & 94,03 a & 9,95 a & $50,94 \mathrm{~b}$ \\
\hline 16 & 3 & $\begin{array}{l}11887,1 \\
\mathrm{C}\end{array}$ & 47,49 c & $9654,1 \mathrm{~d}$ & 78,52 b & 8,44 c & $39,54 \mathrm{c}$ \\
\hline
\end{tabular}




\begin{tabular}{l|l|l|l|l|l|l|l}
\hline 22 & 3 & $\begin{array}{l}15571,1 \\
\mathrm{~b}\end{array}$ & $48,49 \mathrm{c}$ & $12728,4 \mathrm{~b}$ & $82,11 \mathrm{a}$ & $9,21 \mathrm{~b}$ & $48,64 \mathrm{~b}$ \\
\hline 23 & 3 & $\begin{array}{l}18852,0 \\
\mathrm{a}\end{array}$ & $53,64 \mathrm{a}$ & $12500,7 \mathrm{~b}$ & $90,62 \mathrm{a}$ & $10,65 \mathrm{a}$ & $58,77 \mathrm{a}$ \\
\hline Média & - & 11623,5 & 43,14 & 10340 & 76,99 & 8,22 & 40,47 \\
\hline
\end{tabular}

Em que: Médias seguidas por mesma letra na coluna não diferem entre si conforme o teste Scott-knott em 5\% de probabilidade de erro. Valores médios informados em MPa.

Os valores médios de propriedades mecânicas contidos na Tabela 6 são semelhantes aos obtidos em estudos antecedentes. Como no de BOTREL et al. [5], que avaliando clones híbridos do gênero Eucalyptus aos 6,5 anos de idade, encontraram valores médios para o Ec de 7.916,7 MPa e para o fc de 54,04 MPa. Já CAIXETA et al. [6], examinando clones de Eucalyptus spp., obtiveram valores médios para o Ec de 10.164,9 MPa e fc de 61,5 MPa. Portanto, verificou-se que os valores para o Ec do presente estudo se mostraram superiores quando comparados a esses estudos citados e inferiores para fc.

No ensaio mecânico de flexão estática, os valores médios encontrados para os clones de Eucalyptus spp. foram de 10.340 MPa para o MOE e de 76,99 MPa para o MOR, os quais corroboram com os valores médios obtidos por ROSSO [19] ao analisar propriedades mecânicas de Eucalyptus grandis, aos 27 anos de idade, obtendo 10.502,04 MPa para o MOE e 66,64 MPa para o MOR. Nesse contexto, o clone 1 (E. urophylla x E. maidenii) apresentou os maiores valores, tanto para o MOE (16.368,7 MPa) quanto para o MOR (97,63 MPa).

Em sua pesquisa com a madeira de Eucalyptus saligna, aos 8 anos de idade, HASELEIN et al. [20] obtiveram valores médios para o MOE de 9.450 MPa e para o MOR de 73,57 MPa. Já SCANAVACA JUNIOR e GARCIA [21], estudando Eucalyptus urophylla, aos 19 anos, obtiveram valores médios para o MOE de 18.087,6 MPa e para o MOR de 129,1 MPa, evidenciando valores maiores que os encontrados nesse estudo. Da mesma forma, CAIXETA et al. [6] obtiveram elevados valores para MOE (19.184,3 MPa) e para MOR (123,4 MPa) mais elevados do que os do presente estudo.

Para os ensaios de cisalhamento e Dureza Janka foram encontrados valores médios de 8,22 e 40,47 MPa, respectivamente. O clone 23 (E. saligna x E. saligna) se destacou por apresentar os maiores valores, os quais foram de 10,65 e 58,77 MPa, respectivamente. No estudo de LIMA et al. [22], com híbridos de E. grandis x E. urophylla de 8,5 anos de idade, foram encontrados valores de 54,33 MPa para a Dureza Janka, o qual denotou-se semelhante ao valor obtido no presente estudo. Porém, CAIXETA et al. [6] obtiveram valores de 83,4 MPa para Dureza Janka, sendo esses superiores aos valores médios apresentados na Tabela 6.

Contudo, as diferenças encontradas para as propriedades mecânicas quando comparadas dentro desse estudo, bem como com estudos antecedentes estão provavelmente relacionadas às fatores genéticos, idade de corte, bem como fatores ambientais existentes entre os materiais estudados [16, 17, 23].

\subsection{Tensão de crescimento longitudinal}

O valor médio obtido para a DRL foi de 0,147 mm, sendo que o clone 27 (E. urophylla x E. grandis) apresentou o maior valor entre os clones estudados, seguido pelos clone 1 (E. urophylla $\mathrm{x}$ E. maidenii), 7 (E. urophylla $x$ E. grandis), 10 (E. urophylla $x$ E. grandis), 11 (E. urophylla x E. grandis), 25 (E. urophylla x E. grandis) e 73 (E. urophylla $x$ E. globulus). Esse resultado indica do ponto de vista genealógico que as espécies E. grandis e E. urophylla, além de estarem associadas aos menores valores de ppond, também encontram-se vinculados aos maiores níveis de DRL (Tabela 7). 
Tabela 7: Tensões de crescimento longitudinais determinadas pelo módulo de elasticidade dinâmico (TCL1) pelo módulo de elasticidade estático (TCL2), para os clones de Eucalyptus spp.

\begin{tabular}{|c|c|c|c|c|c|c|}
\hline Clones & Grupos & $\mathrm{DRL}(\mathrm{mm})$ & $\mathrm{Ed}_{\mathrm{S}}(\mathrm{MPa})$ & $\begin{array}{l}\mathrm{MOE}_{\mathrm{S}} \\
(\mathrm{MPa})\end{array}$ & $\mathrm{TCL}_{1}(\mathrm{MPa})$ & $\mathrm{TCL}_{2}(\mathrm{MPa})$ \\
\hline 8 & 1 & 0,103 a & 10577,4 & 10472,0 & 24,15 a & 23,91 a \\
\hline 9 & 1 & 0,127 a & 8086,2 & 9260,4 & 22,82 a & $26,13 \mathrm{~b}$ \\
\hline 12 & 1 & 0,113 a & 9185,9 & 8333,7 & 23,13 a & 20,98 a \\
\hline 26 & 1 & $0,121 \mathrm{a}$ & 8528,9 & 8717,4 & 23,01 a & $23,52 \mathrm{a}$ \\
\hline 29 & 1 & $0,161 \mathrm{~b}$ & 10058,9 & 10316,4 & $35,97 \mathrm{~b}$ & $36,89 \mathrm{c}$ \\
\hline 31 & 1 & 0,103 a & 9247,6 & 10588,7 & 21,06 a & 24,11 a \\
\hline 33 & 1 & $0,144 \mathrm{~b}$ & 11142,6 & 9577,6 & $35,60 \mathrm{~b}$ & $30,61 \mathrm{~b}$ \\
\hline 34 & 1 & $0,120 \mathrm{a}$ & 9600,0 & 8772,5 & 25,31 a & 23,13 a \\
\hline 35 & 1 & 0,119 a & 10326,0 & 8582,8 & 27,53 a & 22,88 a \\
\hline 53 & 1 & 0,113 a & 8707,8 & 9809,6 & 21,78 a & 24,53 a \\
\hline 54 & 1 & $0,110 \mathrm{a}$ & 9467,9 & 7658,0 & 23,19 a & 18,76 a \\
\hline 64 & 1 & $0,149 \mathrm{~b}$ & 9564,7 & 8712,2 & $31,68 \mathrm{~b}$ & $28,85 \mathrm{~b}$ \\
\hline 68 & 1 & 0,095 a & 8274,3 & 11189,2 & $17,44 \mathrm{a}$ & 23,59 a \\
\hline 72 & 1 & $0,094 \mathrm{a}$ & 9853,9 & 6832,8 & 20,52 a & 14,23 a \\
\hline 73 & 1 & $0,231 \mathrm{c}$ & 9149,4 & 9602,7 & $46,96 \mathrm{~d}$ & $49,29 \mathrm{~d}$ \\
\hline 1 & 2 & $0,198 \mathrm{c}$ & 12054,4 & 8758,5 & 53,03 e & $38,53 \mathrm{c}$ \\
\hline 3 & 2 & 0,127 a & 9798,9 & 9908,4 & 27,65 a & $27,96 \mathrm{~b}$ \\
\hline 10 & 2 & $0,221 \mathrm{c}$ & 9747,0 & 9691,7 & $47,93 \mathrm{~d}$ & $47,67 \mathrm{~d}$ \\
\hline 11 & 2 & 0,202 c & 9120,3 & 7764,8 & 40,85 c & 34,77 c \\
\hline 13 & 2 & 0,123 a & 8864,2 & 9372,1 & 24,31 a & $25,71 \mathrm{~b}$ \\
\hline 14 & 2 & $0,147 \mathrm{~b}$ & 9512,5 & 9378,9 & $31,08 \mathrm{~b}$ & $30,65 \mathrm{~b}$ \\
\hline 21 & 2 & $0,147 \mathrm{~b}$ & 9451,4 & 8716,9 & $30,86 \mathrm{~b}$ & $28,46 \mathrm{~b}$ \\
\hline 24 & 2 & $0,137 \mathrm{~b}$ & 11274,0 & 11675,6 & $34,32 \mathrm{~b}$ & $35,54 \mathrm{c}$ \\
\hline 25 & 2 & $0,164 \mathrm{c}$ & 9917,0 & 7449,7 & $36,05 \mathrm{~b}$ & $27,08 \mathrm{~b}$ \\
\hline 27 & 2 & $0,316 \mathrm{~d}$ & 9825,7 & 8527,3 & $69,46 \mathrm{f}$ & $60,28 \mathrm{e}$ \\
\hline 7 & 3 & $0,180 \mathrm{c}$ & 9741,8 & 10549,5 & $39,00 \mathrm{c}$ & $42,24 \mathrm{c}$ \\
\hline 16 & 3 & 0,100 a & 9868,9 & 10173,3 & 21,98 a & 22,66 a \\
\hline 22 & 3 & $0,145 \mathrm{~b}$ & 10515,6 & 11539,9 & $33,95 \mathrm{~b}$ & $37,26 \mathrm{c}$ \\
\hline 23 & 3 & $0,140 \mathrm{~b}$ & 12244,9 & 9014,6 & $38,10 \mathrm{c}$ & $28,05 \mathrm{~b}$ \\
\hline Média & - & 0,147 & 9782,3 & 9335,8 & 32,11 & 30,35 \\
\hline
\end{tabular}

Em que: DRL= valor médio entre as posições cardinais de deformação residual longitudinal; EdS = módulo de elasticidade dinâmico na condição saturada; MOES = módulo de elasticidade a flexão estática na condição saturada. Médias seguidas por mesma letra na coluna não diferem entre si em 5\% de probabilidade de erro conforme o teste Scott Knott.

A razão entre os valores médios de módulos de elasticidades dinâmico e estático (EdS/MOES) e consequentemente entre as TCL's obtidas por meio desses (TCL1/TCL2), a qual foi de 1,06, encontram-se dentro da faixa estipulada pela literatura, a qual é de 1,06 a 1,38 [24, 25].

Os clones 68 (E. urophylla x E. globulus) e 72 (E. urophylla x E. globulus) se sobressaíram aos demais clones do estudo, por apresentarem os menores valores para DRL (0,095 e 0,094 mm). Dessa maneira, esses clones podem ser recomendados para programas de melhoramento florestal, visando à produção de madeira sólida. Por outro lado, o clone 27 (E. urophylla x E. grandis) mostrou a maior DRL do estudo e, logo, os maiores valores de TCL1 e TCL2 (69,46 e 60,28 MPa). 
Em sua publicação, TRUGILHO [3], estudando a estimativa da TCL para clones de Eucalyptus dunni aos 8 anos, obteve valor médio de 36,81 MPa para a TCL obtida por meio do módulo de elasticidade ao ensaio de tração paralela as fibras. Já em clones mais velhos, de 13, 15 e 19 anos, o mesmo autor obteve valores médios de 54,42, 64,75 e 60,21 MPa para a TCL, respectivamente. Diante desses resultados, observa-se que os valores médios obtidos para a estimativa da TCL do presente estudo são inferiores aos resultados relatados pelo autor mencionado. Essa inferioridade se deve ao tipo ensaio mecânico proposto para a estimativa da TCL, já que os valores obtidos para o MOE ao ensaio de tração paralela são naturalmente mais elevados do que para o ensaio de flexão estática realizado no presente estudo.

Nesse mesmo trabalho supracitado, TRUGILHO [3] determinou a TCL por meio do Ed de ondas de tensão (stress wave timer) e encontrou valores médios de 7141,7 MPa e para a TCL valor médio de 15,32 MPa. Já em clones com maiores idades, 13, 15 e 19 anos, obteve valores médios para o Ed de 8704,8, 10658,1 e 8291,0 MPa, e para a TCL, valores médios de 22,04, 26,45 e 23,34 MPa, respectivamente. Resultados que denotaram-se inferiores aos do presente estudo. Contudo, deve ser levado em consideração que no estudo de TRUGILHO [3], o método de determinação do módulo de elasticidade, as espécies estudadas, as idades e locais de avaliação não foram os mesmos utilizados no presente estudo, de modo que esses fatores possivelmente colaboraram para essa diferença.

Os clones pertencentes ao grupo 1 demonstraram os menores valores de TCL (26,09 MPa), corroborando com os valores apresentados para a DRL na forma de grupos. Já os valores médios obtidos para os clones pertencentes aos grupos 2 (35,6 MPa) e 3 (33,21 MPa), apresentaram as maiores estimativas para a TCL2.

\subsection{Correlações entre as variáveis do estudo}

Através da análise de Pearson apresentada na Tabela 8, pode-se observar que a DRL apresentou correlações positivas e significativas com o MOE obtido pelo ensaio de flexão estática. Já a ppond não apresentou correlações com a DRL e TCL2, indicando baixa influência sobre essas propriedades.

Tabela 8: Correlação de Pearson entre as variáveis do estudo.

\begin{tabular}{l|l|l|l|l|l|l|l|l|l}
\hline & DRL & TCL $_{2}$ & $\rho_{\text {pond }}$ & Ec & fc & MOE & MOR & Cisa & Dureza \\
\hline DRL & 1 & $0,808^{*}$ & $-0,009^{\mathrm{NS}}$ & $-0,001^{\mathrm{NS}}$ & $-0,124^{\mathrm{NS}}$ & $0,274^{*}$ & $0,068^{\mathrm{NS}}$ & $-0,034^{\mathrm{NS}}$ & $-0,068^{\mathrm{NS}}$ \\
\hline $\mathrm{TCL}_{2}$ & & 1 & $0,143^{\mathrm{NS}}$ & $0,033^{\mathrm{NS}}$ & $-0,005^{\mathrm{NS}}$ & $0,354^{*}$ & $0,255^{*}$ & $0,072^{\mathrm{NS}}$ & $0,109^{\mathrm{NS}}$ \\
\hline IRT & & & $-0,176^{\mathrm{NS}}$ & $-0,055^{\mathrm{NS}}$ & $-0,264^{*}$ & $0,023^{\mathrm{NS}}$ & $-0,107^{\mathrm{NS}}$ & $-0,211^{\mathrm{NS}}$ & $-0,166^{\mathrm{NS}}$ \\
\hline$\rho_{\text {pond }}$ & & & 1 & $0,636^{*}$ & $0,876^{*}$ & $0,760^{*}$ & $0,801^{*}$ & $0,881^{*}$ & $0,875^{*}$ \\
\hline Ec & & & & 1 & $0,743^{*}$ & $0,615^{*}$ & $0,529^{*}$ & $0,635^{*}$ & $0,652^{*}$ \\
\hline fc & & & & & 1 & $0,745^{*}$ & $0,739^{*}$ & $0,799^{*}$ & $0,802^{*}$ \\
\hline MOE & & & & & & 1 & $0,743^{*}$ & $0,640^{*}$ & $0,634^{*}$ \\
\hline MOR & & & & & & & 1 & $0,670^{*}$ & $0,707^{*}$ \\
\hline Cisa & & & & & & & & 1 & $0,838^{*}$ \\
\hline Dureza & & & & & & & & & 1 \\
\hline
\end{tabular}

Em que: TCL2 = tensão de crescimento longitudinal a 12\% de umidade $\left(\mathrm{kgf} / \mathrm{cm}^{2}\right)$; DRL = deformação residual longitudinal; $\rho$ pond $=$ massa específica básica ponderada $\left(\mathrm{g} / \mathrm{cm}^{3}\right) ; \mathrm{Ec}=$ módulo de elasticidade a compressão paralela às fibras $\left(\mathrm{kgf} / \mathrm{cm}^{2}\right) ; \mathrm{fc}=$ resistência a compressão paralela às fibras $\left(\mathrm{kgf} / \mathrm{cm}^{2}\right) ; \mathrm{MOE}=$ módulo de elasticidade a flexão estática $\left(\mathrm{kgf} / \mathrm{cm}^{2}\right) ; \mathrm{MOR}=$ módulo de ruptura a flexão estática $\left(\mathrm{kgf} / \mathrm{cm}^{2}\right) ;$ Cisa = resistência ao cisalhamento $\left(\mathrm{kgf} / \mathrm{cm}^{2}\right) ;$ Dureza = Dureza Janka $\left(\mathrm{kgf} / \mathrm{cm}^{2}\right) ; *$ = significativo em 5\% probabilidade de erro; NS = não significativo.

Analogamente, outros estudos analisando a madeira de clones de Eucalyptus, não encontraram correlações significativas entre a DRL e a massa específica básica [7, 23]. Porém, a ppond apresentou correlações significativas e positivas com todas as propriedades mecânicas.

Em seu estudo, HASELEIN et al. [20] confirmaram que a redução da massa específica ocasionou uma redução proporcional nas propriedades mecânicas. No estudo de SILVA [16] com a madeira de Eucalyptus grandis aos 10, 14, 20 e 25 anos de idade, houve correlação positiva entre as propriedades de resistência e rigidez mecânica da madeira (MOR e MOE) e a massa específica aparente a 12\% de umidade, corroborando com as correlações do presente estudo. 
No estudo de LIMA et al. [22], analisando cada clones do gênero Eucalyptus isoladamente, os autores não encontraram correlação entre a DRL e algumas propriedades físicas (massa específica básica e contração volumétrica) e mecânicas (Dureza Janka, compressão paralela as fibras, MOE e MOR a flexão estática) da madeira, exceto para o MOE a flexão estática de um clone hibrido natural de Eucalyptus grandis W Hill. ex Maiden de 15 anos de idade.

Para ADORNO e GARCIA [23], que estudaram as correlações das propriedades da madeira de clones de Eucalyptus grandis e Eucalyptus urophylla, ambos com 8 anos de idade, foi observado que a massa específica básica apresentou correlação positiva com todas as propriedades mecânicas (resistência ao cisalhamento radial, resistência ao cisalhamento tangencial, resistência à compressão paralela às fibras, módulo de elasticidade na flexão estática, resistência à flexão estática e resistência à tração paralela às fibras) avaliadas em laboratório para ambas as espécies.

Portanto, os resultados obtidos e discutidos no presente estudo denotam a grande variabilidade das propriedades físicas e mecânicas da madeira de clones do gênero Eucalyptus, o que indica a importância de serem ostensivamente caracterizadas a fim de que sejam levadas em consideração sinergicamente na qualificação dessas madeiras com vistas à confecção de produtos sólidos.

\section{CONCLUSÕES}

- Os clones 1 (Eucalyptus urophylla x Eucalyptus maidenii), 23 (Eucalyptus saligna x Eucalyptus saligna) e 24 (Eucalyptus saligna x Eucalyptus saligna) se destacaram por apresentarem maiores valores para a massa específica básica e ponderada, como também maiores valores para as propriedades mecânicas;

- Os valores médios de tensão de crescimento longitudinal obtida pelo módulo de elasticidade dinâmico foram superiores a os estimados com a tensão de crescimento longitudinal obtida pelo módulo de elasticidade estático;

- Os clones 68 e 72, ambos de genealogia Eucalyptus urophylla x Eucalyptus globulus, se destacaram com os menores níveis de deformação residual longitudinal, dessa maneira podem ser indicados para um melhoramento genético visando menores níveis de defeitos oriundos das tensões de crescimento;

- A massa específica básica ponderada e as propriedades mecânicas não apresentaram correlações com a deformação residual longitudinal e consequentemente com a tensão de crescimento longitudinal, podendo ser desconsideradas em um programa de melhoramento genético, quando a seleção tem por finalidade a busca por clones com menor propensão a defeitos oriundos das tensões de crescimento.

\section{AGRADECIMENTOS}

Ao Conselho Nacional de Desenvolvimento Científico e Tecnológico, pelas bolsas de estudo e de produtividade em pesquisa; e à empresa CMPC Celulose Riograndense, pelo apoio e assistência técnica para a realização do trabalho.

\section{BIBLIOGRAFIA}

[1] ROCHA, M.P., Eucalyptus grandis Hill ex Maiden e Eucalyptus dunnii Maiden como fontes de matériaprima para serrarias, Tese de D.Sc., Universidade Federal do Paraná, Curitiba, 2000.

[2] AGUIAR, O.J.R., Método para controle das rachaduras de topo em toras de Eucalyptus grandis W. Hill. Ex Maiden, visando a produção de lâminas por desenrolamento, Dissertação de M.Sc, Escola Superior de Agricultura "Luiz de Queiroz", Piracicaba, 1986.

[3] TRUGILHO, P.F., Tensão de crescimento em árvores vivas de clones de Eucalyptus spp. e de Eucalyptus dunnii Maiden e propriedades de sua madeira, Tese de Pós- D.Sc, Universidade Federal do Paraná, Curitiba 2005.

[4] NICHOLSON, J. E., "Growth stresses differences in Eucalyptus”, Forest Science, v. 9, n. 3, pp.169, 1973.

[5] BOTREL, M.C.G, SILVA, J. R.M., TRUGILHO, P.F., et al. "Ganho genético em propriedades físicas e mecânicas de clones de Eucalyptus”, Scientia Forestalis, v. 76, n. 4, pp. 13-19, 2007.

[6] CAIXETA, R.P., TRUGILHO, P.F., ROSADO, S.C.S., et al. "Propriedades e classificação da madeira aplicadas à seleção de genótipos de Eucalyptus” Árvore, v. 27, n. 1, pp. 43-51, 2003. 
[7] BELTRAME, R., LAZAROTTO, M., HASELEIN, C. R., et al. "Determinação das deformações residuais longitudinais decorrentes das tensões de crescimento em Eucalyptus spp”, Ciência Florestal, v. 22, n. 2, pp. 343-351, 2012.

[8] BARROSO, L.P.; ARTES, R., Análise multivariada Análise multivariada. Lavras, UFLA, 2003.

[9] MINGOTI, S.A., Análise de dados através de métodos de estatística multivariada, Belo Horizonte, UFMG, 2005.

[10] VITAL, B.R., “Métodos de determinação da densidade da madeira”, SIF/UFV Boletim Técnico, v. 1, n. 1, pp. 1-21, 1984.

[11] AMERICAN SOCIETY FOR TESTING AND MATERIALS, "Standard Test methods for small clear specimens of timber”, ASTM D143-94, West Conshohocken, 2007.

[12] BODIG, J., JAYNE, B., Mechanics of wood and wood composite, Nova York, Van Nostrand Reinhold Company Inc., 1982.

[13] LIMA, J.T., SARTORIO, R.C., TRUGILHO, P.F., et al. "Uso do resistógrafo para estimar a densidade básica e a resistência à perfuração da madeira de Eucalyptus.”, Scientia Forestalis, v. 75, n. 3, pp. 85-93, 2007.

[14] TREVISAN, R., ELOY, E., DENARDI, L., et al. "Variação axial e efeito do desbaste na massa específica das árvores centrais de Eucalyptus grandis”, Ciência Rural, v. 42, n. 2, pp. 312-318, 2012.

[15] TREVISAN, R. DENARDI, L., HASELEIN, C. R., et al. "Efeito do desbaste e variação longitudinal da massa específica básica da madeira de Eucalyptus grandis”, Scientia Forestalis, v. 95, n. 3, pp. 393-399, 2012.

[16] SILVA, J.C., Caracterização da madeira de Eucalyptus grandis Hill ex Maiden, de diferentes idades, visando a sua utilização na indústria moveleira, Dissertação de D.Sc., Universidade Federal do Paraná, Curitiba, 2002.

[17] ALZATE, S. B. A., TOMAZELLO FILHO, M., PIEDADE, S.M.S. "Variação longitudinal da densidade básica da madeira de clones de Eucalyptus grandis Hill ex Maiden, Eucalyptus saligna Sm. e Eucalyptus grandis x Eucalyptus urophylla.” Scientia Forestalis, n. 68, n. 2, pp.87-95, 2005.

[18] TREVISAN, R., Efeito da intensidade de desbaste nas características dendrométricas e tecnológicas de Eucalyptus grandis Hill ex Maiden. Dissertação de M.Sc., Universidade Federal de Santa Maria, Santa Maria, 2006.

[19] ROSSO, S. Predição das propriedades de Eucalyptus grandis Maiden por espectroscopia no infravermelho próximo. Tese de D.Sc., Universidade Federal do Paraná, Curitiba, 2010.

[20] HASELEIN, C.R., BERGER, R., GOULART, M., et al. "Propriedades de flexão estática da madeira úmida e a $12 \%$ de umidade de um clone de Eucalyptus saligna Smith sob o efeito do espaçamento e da adubação”, Ciência Florestal, v. 12, n. 2, pp.147-152, 2002.

[21] SCANAVACA JUNIOR, L.; GARCIA, J. N. "Determinação das propriedades físicas e mecânicas da madeira de Eucalyptus urophylla”, Scientia Forestalis, v. 65, n. 1, pp. 120-129, 2004.

[22] LIMA, J.T., TRUGILHO, P.F., ROSADO, S.C.S., et al. "Deformações residuais decorrentes de tensões de crescimento em eucaliptos e suas associações com outras propriedades”, Árvore, v. 28, n. 1, pp. 107-116, 2004.

[23] ADORNO, M. C., GARCIA, J. N. “Correlações lineares entre as principais características tecnológicas da madeira de clones de Eucalyptus grandis e Eucalyptus urophylla”, Scientia Forestalis, v. 63, n. 1, pp. 4453, 2003.

[24] STANGERLIN, D.M., CALEGARI, L., SANTINI, E.J., et al. "Determinação do módulo de elasticidade em madeiras por meio de métodos destrutivo e não-destrutivo”, Revista Brasileira de Ciências Agrárias, v. 3, n. 2, pp. 145-150, 2008.

[25] HASEGAWA, M., TAKATA, M., MATSUMURA, J., et al. "Effect of wood properties on within-tree variation in ultrasonic wave velocity in softwood”, Ultrasonics, v. 51, n. 3, pp. 296-302, 2011. 